Ana Luiza de Araújo Campos'

Maria Alix Leite Araújo'

Simone Paes de Melo

Roumayne Fernandes Vieira AndRade'

Marcelo luiz Carvalho Gonçalves ${ }^{2}$

\title{
Sífilis em parturientes: aspectos relacionados ao parceiro sexual
}

\author{
Syphilis in parturients: aspects related to the sexpartner
}

Artigo Original

Palavras-chave

Sífilis

Sífilis congênita

Parceiros sexuais

Gestantes

Doenças sexualmente transmissíveis

Keywords

Syphilis

Syphilis, congenital Sexual partners

Pregnant women Sexually transmitted diseases

\section{Resumo}

OBJETIVOS: Analisar o perfil sociodemográfico e comportamental dos parceiros sexuais, a proporção daqueles inadequadamente tratados e os motivos da não realização do tratamento. MÉTODOS: Estudo quantitativo cuja coleta de dados ocorreu de maio a outubro de 2008, em cinco maternidades públicas de Fortaleza, Ceará. Foram aplicados questionários às parturientes internadas com sífilis que informaram ter parceiro sexual fixo. Foram analisadas as variáveis sociodemográficas e as relacionadas à comunicação, diagnóstico e tratamento dos parceiros sexuais. Os dados foram digitados no programa Statistical Package for the Social Sciences e foram analisados por meio de distribuições de frequências, de medidas de tendência central e de dispersão. RESULTADOS: Participaram do estudo 56 parturientes. Os parceiros sexuais tinham média de idade de 29 anos, menos de 7 anos de estudo (50\%), atividade laboral $(82,1 \%)$, renda familiar inferior a 1 salário-mínimo (6,4\%). Eram o pai da criança 92,9 e 69,6\% moravam com a parturiente. Faziam uso de álcool e drogas 50 e 12\%, respectivamente. Foram comunicados do diagnóstico $75,0 \%$ parceiros, a comunicação foi feita pela própria mulher em $78,6 \%$ casos e ficaram sabendo do resultado do exame de VDRL antes ou durante o pré-natal, 59,5\%. Não revelaram o diagnóstico 25,0\% mulheres e os motivos alegados foram: desconhecer a importância do tratamento do parceiro $(50,0 \%)$, não ter estado com este parceiro após o diagnóstico $(42,9 \%)$ e estar brigada $(7,1 \%)$. Dos que souberam do diagnóstico antes ou durante o pré-natal, $56,0 \%$ foram tratados e $6(42,8 \%)$ foram considerados adequadamente tratados. Dentre os que não receberam tratamento, $63,6 \%$ se recusaram por não se sentir doentes, não acreditar no tratamento e medo de injeção. CONCLUSÕES: Os parceiros são comunicados do diagnóstico de sífilis da gestante; entretanto, poucos são adequadamente tratados.

\section{Abstract}

PURPOSES: To analyze the sociodemographic and behavioral profile of sex partners, the proportion of those inadequately treated as well as to verify how many of them were inadequately treated and why some were not treated. METHODS: Quantitative study with data collected from May to October, 2008 at five public maternities in Fortaleza, Ceará. A survey was carried out with parturients who were hospitalized with syphilis and had a stable sex partner. We analyzed sociodemographic variables and those related to communication, diagnosis and treatment of sex partners. The data were entered into the Statistical Package for the Social Sciences and were analyzed using frequency distributions, measures of central tendency and dispersion. RESULTS: The study included 56 pregnant women. Most sexual partners were young adults aged on average 29 years, 50\% of them had studied for less than seven years, 82.1 worked and $46.4 \%$ had a family income of less than a minimum wage. Of all the partners, $92.9 \%$ were the child's father and $69.6 \%$ lived with the women. Fifty percent and $12 \%$ were alcohol and drug users, respectively. Most partners (75.0\%) were told about the diagnosis by the women, and in $78.6 \%$ of cases they were aware of the VDRL result before or during the prenatal period. However, $25.0 \%$ of the women did not communicate the result to their partners for the following reasons: not knowing the importance of the partner's treatment $(50.0 \%)$, not being together after the diagnosis $(42.9 \%)$ and having a quarrel $(7.1 \%)$. Of the partners who were informed about the result before or during the prenatal period, $56.0 \%$ were treated and six $(42.8 \%)$ were considered to have been
Correspondência

Maria Alix Leite Araúio Universidade de Fortaleza - UNIFOR Avenida Washington Soares, 1321 - Edson Queiroz CEP: 60811 1-905 Fortaleza (CE), Brasil

Recebido

$03 / 05 / 2012$

Aceito com modificações
Trabalho realizado no Instituto de Pesquisa Clínica Evandro Chagas, Fundação Oswaldo Cruz - IPEC/FIOCRUZ - Rio de Janeiro (RJ), Brasil.

'Programa de Pós-graduação em Saúde Coletiva, Universidade de Fortaleza - UNIFOR - Fortaleza (CE), Brasil. ${ }^{2}$ Instituto de Pesquisa Clínica Evandro Chagas, Fundação Oswaldo Cruz - IPEC/FIOCRUZ - Rio de Janeiro (RJ), Brasil. 
properly treated. Among the ones who did not receive treatment, 63.6\% refused it because they did not feel sick, because they did not believe in the treatment and because they were afraid of injections. CONCLUSIONS: Partners are told about the syphilis diagnosis of the pregnant women; however, only a few are properly treated.

\section{Introdução}

A sífilis congênita (SC) é a infecção do concepto pelo Treponema pallidum por via transplacentária, a partir da mãe infectada não tratada ou inadequadamente tratada. A transmissão pode ocorrer em qualquer fase da gestação ou durante o parto ${ }^{1}$, com uma taxa que varia de 30 a $100 \%$ dependendo do estágio da sífilis na mãe $e^{2}$. A infecção é a principal causa de óbito fetal na presença da sífilis materna ${ }^{3}$. Embora os registros de SC sejam muito dependentes da qualidade das notificações e dos sistemas de vigilância epidemiológica de estados e municípios, os dados oficiais no Brasil mostram que entre os anos de 2000 a 2009 ocorreram 52.541 casos, com incidência bastante variável entre as diferentes regiões. No Ceará, nesses mesmos anos foram registrados 2.747 casos com taxa de incidência que variou de 0,1 a 4,7 a casos por 1.000 nascidos vivos ${ }^{4}$.

A possibilidade de erradicação da SC encontra-se no diagnóstico precoce e tratamento adequado da sífilis gestacional que, embora de fácil diagnóstico, tratamento barato e eficaz, ainda se mantêm como um grave problema de saúde pública, principalmente nos países em desenvolvimento ${ }^{2,5,6}$.

O Ministério da Saúde preconiza que durante a assistência pré-natal toda gestante seja submetida a pelo menos dois exames de Venereal Disease Research Laboratory (VDRL), um por ocasião da primeira consulta e outro por volta da $30^{a}$ semana gestacional. Deve-se ainda realizar um VDRL no momento do parto para garantir ao recém-nascido a possibilidade de tratamento precoce, caso a gestante não tenha sido tratada ou tenha se reinfectado após o tratamento ${ }^{7}$. São consideradas adequadamente tratadas as gestantes que tenham sido medicadas com penicilina $\mathrm{G}$ benzatina, com dosagem adequada à fase clínica da doença, cujo tratamento tenha sido concluído pelo menos 30 dias antes do parto e que seu parceiro tenha sido concomitantemente tratado ${ }^{2}$.

No Brasil, estudos mostram que a falta de tratamento dos parceiros sexuais ${ }^{8}$ é um dos principais entraves para o controle da SC. No ano de 2006, dentre os casos notificados de SC, havia informações sobre o tratamento dos parceiros sexuais em apenas $13,2 \%{ }^{4}$, situação também encontrada em Salvador, Bahia, na qual um estudo mostrou 96,2\% de parceiros das gestantes com sífilis sem tratamento adequado ${ }^{9}$, em Campos, Rio de Janeiro ${ }^{10}$ e Campo Grande no Mato Grosso do Sul ${ }^{11}$ cujas proporções foram de 88 e $60 \%$, respectivamente.
Em vista da importância do tratamento adequado do parceiro das gestantes com VDRL reagente e a escassez de informações sobre esse importante elo na cadeia de transmissão da infecção, este estudo teve por objetivo analisar o perfil sociodemográfico e comportamental dos parceiros de gestantes com VDRL reagente identificando os motivos do não tratamento.

\section{Métodos}

Estudo transversal e descritivo, realizado a partir de uma pesquisa que teve por objetivo avaliar aspectos sociodemográficos e epidemiológicos de parturientes com exame de VDRL reagente, em Fortaleza, Ceará. Neste artigo são mostradas as informações referentes aos parceiros sexuais, fornecidas pelas parturientes que apresentaram VDRL reagente no período gestacional ou no momento do parto.

A coleta de dados foi realizada no período de maio a outubro de 2008, em cinco maternidades públicas municipais, referência na área de Obstetrícia para parturientes de alto risco no município de Fortaleza e cidades do interior do Estado do Ceará. Alunas do curso de Enfermagem devidamente treinadas aplicaram um questionário, previamente testado, às parturientes que se encontravam no alojamento conjunto e tinham sido diagnosticadas com sífilis. Visando garantir o sigilo e a tranquilidade das pacientes em fornecer as informações, estas foram levadas para um espaço reservado para aplicação dos questionários.

Foram avaliadas as variáveis dos parceiros sexuais cujos dados foram complementados com as informações disponíveis no cartão da gestante e nos prontuários médicos. A entrevista ocorreu com as parturientes que referiram estar com parceiro sexual fixo na ocasião da entrevista, mesmo na eventualidade de este não ser o pai do recém-nascido. Como parceiros sexuais fixos foram considerados todos aqueles que mantinham um relacionamento sexual com a parturiente há pelo menos três meses, independentemente de morarem juntos. Esse período considerou o tempo necessário recomendado pelo Ministério da Saúde para tratamento dos parceiros sexuais de pessoas com doenças sexualmente transmissíveis ${ }^{1}$.

As variáveis analisadas foram: sociodemográficas (idade, escolaridade em anos de estudo, ocupação, renda familiar, tempo de relacionamento, se mora com a parturiente e se é o pai do recém-nascido); e relacionadas à comunicação, diagnóstico e tratamento dos parceiros sexuais (se o parceiro foi comunicado do diagnóstico, momento da comunicação, quem comunicou, motivo alegado pela parturiente para não 
ter comunicado o diagnóstico, se ela sofreu algum tipo de violência após a revelação do diagnóstico, se o parceiro foi tratado e, caso sim, se o tratamento foi adequado).

Os dados foram tabulados e analisados no programa Statistical Package for the Social Sciences (SPSS). Foi realizada análise univariada por meio de distribuições de frequências ou, quando aplicável, de medidas de tendência central e de dispersão.

Este estudo foi aprovado pelo Comitê de Ética da Universidade de Fortaleza com o Parecer no 059/2008. As parturientes foram convidadas a participar do estudo, após esclarecimentos sobre os objetivos e os procedimentos a serem utilizados e todas assinaram o termo de consentimento livre e esclarecido.

\section{Resultados}

No período de 6 meses foram entrevistadas 58 parturientes com VDRL reagente, e, destas, $56(96,6 \%)$ referiram parceria sexual fixa na ocasião da pesquisa. Cinco pacientes $(8,9 \%)$ informaram que o parceiro já tinha adquirido sífilis anteriormente, $16(28,6 \%)$ que estes tinham atividades sexuais fora do relacionamento e apenas $8(14,3 \%)$ disseram ter usado preservativo nas relações após o diagnóstico de sífilis. Faziam uso regular de bebidas alcoólicas 28 (50\%), e $7(12,5 \%)$ de drogas ilícitas. Ficaram sabendo do resultado positivo do exame de VDRL ainda durante o pré-natal 29 $(51,8 \%)$ parturientes.

A Tabela 1 apresenta variáveis sociodemográficas e tempo de relacionamento dos parceiros sexuais com a parturiente. Predominou a faixa etária de 21 a 30 anos com $29(51,8 \%)$, e a média da idade dos parceiros foi de 29 anos, variando de 18 a 54 anos (DP $\pm 8,1)$. A metade dos parceiros, 28 (50,0\%), tinha menos de 7 anos de estudo, 46 $(82,1 \%)$ trabalhavam na ocasião da pesquisa e $26(46,4 \%)$ tinham renda familiar inferior a um salário-mínimo. Disseram ter menos de cinco anos de relacionamento 39 $(69,6 \%)$ das parturientes, $39(69,6 \%)$ moravam com o companheiro e o parceiro era o pai do recém-nascido em $52(92,9 \%)$ casos.

Foram comunicados do diagnóstico $42(75,0 \%)$ parceiros; a comunicação foi feita pela própria mulher em $33(78,6 \%)$ casos e ficaram sabendo do resultado do exame de VDRL antes ou durante o pré-natal $25(59,5 \%)$. Não revelaram o diagnóstico $14(25,0 \%)$ mulheres e os motivos alegados foram: desconhecer a importância do tratamento do parceiro $(50,0 \%)$, não ter estado com com este após o diagnóstico $(42,9 \%)$ e estar brigada $(7,1 \%)$. Relataram ter sofrido algum tipo de violência após a revelação do diagnóstico $(4,7 \%)$.

Das 56 mulheres entrevistadas, $49(87,5 \%)$ foram informadas da importância do tratamento dos parceiros e $14(56,0 \%)$ dos que souberam do diagnóstico antes ou durante o pré-natal foram tratados (Tabela 2).
Tabela 1. Variáveis sociodemográficas e tempo de relacionamento dos parceiros sexuais de parturientes com Venereal Disease Research Laboratory reagente

\begin{tabular}{|c|c|c|}
\hline Variáveis & $n$ & $\%$ \\
\hline \multicolumn{3}{|c|}{ Faixa efária (em anos) } \\
\hline $11-20$ & 8 & 14,3 \\
\hline $21-30$ & 29 & 51,8 \\
\hline$>30$ & 19 & 33,9 \\
\hline \multicolumn{3}{|c|}{ Escolaridade (em anos de estudo) } \\
\hline$\leq 3$ & 9 & 16,1 \\
\hline $4-7$ & 19 & 33,9 \\
\hline$>7$ & 17 & 30,3 \\
\hline Ignorado & 11 & 19,6 \\
\hline \multicolumn{3}{|l|}{ Trabalha } \\
\hline $\operatorname{Sim}$ & 46 & 82,1 \\
\hline Não & 10 & 17,9 \\
\hline \multicolumn{3}{|c|}{ Renda familiar (em salários-mínimos) } \\
\hline$<1$ & 26 & 46,4 \\
\hline$>1$ & 13 & 23,2 \\
\hline Ignorado & 17 & 30,4 \\
\hline \multicolumn{3}{|c|}{ Tempo de relacionamento com a gestante (em anos) } \\
\hline$<1$ & 11 & 19,6 \\
\hline $1-5$ & 28 & 50,0 \\
\hline$>5$ & 13 & 23,2 \\
\hline Ignorado & 4 & 7,1 \\
\hline \multicolumn{3}{|c|}{ Mora com a gestante } \\
\hline Sim & 39 & 69,6 \\
\hline Não & 17 & 30,4 \\
\hline \multicolumn{3}{|c|}{ É o pai do recém-nascido } \\
\hline Sim & 52 & 92,9 \\
\hline Não/Ignorado & 04 & 7,2 \\
\hline Total & 56 & 100,0 \\
\hline
\end{tabular}

Tabela 2. Variáveis relacionadas à comunicacão do diagnóstico e ao tratamento dos parceiros sexuais de parturientes com VDRL reagente

\begin{tabular}{|c|c|c|}
\hline Variáveis & $\mathrm{n}$ & $\%$ \\
\hline \multicolumn{3}{|l|}{ Parceiro foi comunicado do diagnóstico $(n=56)$} \\
\hline Sim & 42 & 75,0 \\
\hline Não & 14 & 25,0 \\
\hline \multicolumn{3}{|l|}{ Período da comunicacão ( $n=42)$} \\
\hline Antes/durante o pré-natal & 25 & 59,5 \\
\hline No parto/puerpério atual & 17 & 40,5 \\
\hline \multicolumn{3}{|l|}{ Quem comunicou o diagnóstico ( $n=42)$} \\
\hline Gestante & 33 & 78,6 \\
\hline Profissional de saúde & 7 & 16,7 \\
\hline Ignorado & 2 & 4,7 \\
\hline \multicolumn{3}{|l|}{$\begin{array}{l}\text { Motivo alegado pela parturiente para não comunicar o } \\
\text { diagnóstico ( } n=14)\end{array}$} \\
\hline Estavam brigados & 1 & 7,1 \\
\hline Desconhecia a importância & 7 & 50,0 \\
\hline Não esteve com o parceiro após o diagnóstico & 6 & 42,9 \\
\hline \multicolumn{3}{|c|}{ Parceiro foi violento após comunicação do diagnóstico ( $\mathrm{n}=42$ ) } \\
\hline Sim & 2 & 4,7 \\
\hline Não & 38 & 90,6 \\
\hline Ignorado & 2 & 4,7 \\
\hline \multicolumn{3}{|c|}{ Gestante informada da importância do trotamento do parceiro $(n=56)$} \\
\hline Sim & 49 & 87,5 \\
\hline Não & 5 & 8,9 \\
\hline Ignorado & 2 & 3,6 \\
\hline \multicolumn{3}{|c|}{ Após comunicado do diagnóstico parceiro foi tratado ( $\mathrm{n}=25$ ) } \\
\hline Sim & 14 & 56,0 \\
\hline Não/Ignorado & 11 & $44, \mathrm{C}$ \\
\hline \multicolumn{3}{|l|}{ Adequação do tratamento do parceiro $(n=14)$} \\
\hline $\operatorname{Sim}$ & 6 & 42,8 \\
\hline Não & 8 & 47,2 \\
\hline
\end{tabular}


Pelas informações colhidas, entre os parceiros que receberam tratamento, $6(42,8 \%)$ foram considerados adequadamente tratados. Onze $(44,0 \%)$ não receberam tratamento, destes, sete $(63,6 \%)$ se recusaram (por não se sentirem doentes, não acreditarem no tratamento e medo de injeção), dois tiveram o exame de VDRL negativo, um não fez o exame de VDRL e um alegou não ter tempo.

\section{Discussão}

Existem, na literatura, poucos detalhamentos acerca das questões que envolvem as dificuldades relacionadas ao tratamento dos parceiros sexuais de gestantes com sífilis. Portanto, a compreensão do que ocorre com esses parceiros é de extrema importância para a eliminação da SC, uma vez que esse problema é um dos maiores empecilhos para que a gestante seja considerada adequadamente tratada ${ }^{12}$, tendo como consequência a necessidade de tratamento do bebê, o que pode gerar transtornos para a família. A maior parte dos trabalhos relacionados à sífilis gestacional analisa variáveis das gestantes ${ }^{13,14}$ e as poucas informações referentes aos parceiros limitam-se à adequação ou não do tratamento recebido ${ }^{13}$.

Os parceiros sexuais das parturientes deste estudo na maioria eram jovens, com pouca escolaridade, com atividade laboral e renda familiar inferior a um salário-mínimo. Considerando que $92,9 \%$ dos parceiros era o pai do recém-nascido, $69,6 \%$ moravam com a gestante (casados ou em união estável) e que mais da metade, 59,5\% foram comunicados do resultado do exame de VDRL antes ou durante o pré-natal, fica patente a inoperância dos serviços de saúde e a falta de sensibilidade para a elaboração de estratégias de convocação adaptadas às realidades de vida das mulheres. É sabido que o tratamento do parceiro envolve questões complexas; entretanto, a grande maioria foi comunicada do diagnóstico e pode ser localizada pelo serviço de saúde.

O controle da SC parece que ainda não representa um problema importante a ser enfrentado pelos responsáveis pelas políticas públicas de saúde, considerando que esse problema é antigo ${ }^{15}$ e permanece, ainda na atualidade, sem solução ${ }^{13,16}$. Estudos mostram que é possível evitar a transmissão vertical de doenças quando existe um investimento maciço no seu controle, como é o caso do HIV ${ }^{17}$.

Informaram diagnóstico anterior de sífilis no parceiro $8,9 \%$ das gestantes. Não foi possível identificar nesse estudo se a sífilis foi adquirida durante o relacionamento atual. Vale salientar que 28,6\% delas tinham conhecimento de relações extraconjugais dos parceiros. Esse padrão de comportamento masculino, considerado normal na visão dos homens ${ }^{18}$, dificulta sobremaneira o manejo dos casos, uma vez que o diagnóstico de DST pode desvendar a condição de infidelidade, o que pode ser um impedimento para que os homens se dirijam aos serviços para tratamento.

Mesmo entre as mulheres que tinham conhecimento da atividade sexual do parceiro fora do relacionamento, o uso do preservativo foi referido por apenas oito. Para as mulheres, especialmente aquelas de parceiro fixo, a inserção do preservativo nas relações sexuais é difícil ${ }^{19}$. Em se tratando da presença de uma DST, especialmente da sífilis, essa condição se agrava, devido à possibilidade da transmissão vertical. Não se pode desconsiderar a dificuldade do uso do preservativo, especialmente em relações estáveis; entretanto, deve existir nos serviços de saúde uma postura diferenciada, que favoreça o acolhimento e identificação conjunta com a paciente de estratégias de negociação com o parceiro, uma vez que a reinfecção pode perpetuar a sífilis.

Entre as mulheres que tinham conhecimento anterior da sífilis ou souberam da infecção durante o pré-natal, $86,2 \%$ comunicaram o diagnóstico ao parceiro e, desses, somente $56 \%$ receberam pelo menos uma dose da penicilina benzatina. É preciso reforçar a necessidade de valorização e melhoria da qualidade do aconselhamento em casos de gestantes com sífilis $\mathrm{O}$ aconselhamento, quando bem executado, é um instrumento importante para a quebra da cadeia de transmissão das DST, pois proporciona à pessoa avaliação das condições de risco ${ }^{1}$. Pessoas que recebem o aconselhamento para DST têm maior chance de o parceiro comparecer ao serviço de saúde para tratamento ${ }^{20}$.

A maioria das parturientes $(87,5 \%)$ relatou que foram informadas da importância do tratamento do parceiro, a fim de evitar a transmissão vertical, a reinfecção e garantir a adequação do seu tratamento. Quando analisado o total de parceiros que souberam do diagnóstico de sífilis na gestante antes ou durante o pré-natal, apenas seis (24\%) parceiros realizaram o tratamento adequado ${ }^{2}$. Assim, percebe-se que a maioria dos parceiros não são tratados, o que parece ser um problema vivenciado em diferentes regiões do país ${ }^{8,11,13,21}$.

A dificuldade de tratamento do parceiro sexual de portadores de DST pode estar relacionada à própria construção histórica das políticas de saúde, que sempre foram excludentes em relação ao homem, provocando a baixa procura por atendimento ${ }^{22}$. Pode também estar associada à visão que os homens têm em relação à saúde, pois atribuem à mulher a responsabilidade pelo cuidado ${ }^{23,24}$. Por outro lado, existem ainda questões inerentes aos serviços ${ }^{21,25} \mathrm{e}$ ao comportamento dos parceiros, como é o caso do uso de drogas e álcool ${ }^{26}$.

Duas parturientes revelaram ter sofrido violência física no momento que comunicaram o diagnóstico de sífilis ao parceiro, fato que evidencia a possibilidade de a mulher sofrer violência pela revelação do diagnóstico, situação que não pode ser desconsiderada. No contexto 
das DST, o receio da reação do parceiro está relacionado a não comunicação do diagnóstico ${ }^{27}$, o que pode trazer como consequência falhas no tratamento. Esse achado reforça mais uma vez a necessidade de repensar as ações de aconselhamento desenvolvidas na atenção primária, uma vez que, usualmente, as orientações são realizadas de forma prescritiva e completamente descontextualizada da vida da gestante ${ }^{28}$.

As informações referentes aos parceiros sexuais, por terem sido fornecidas pelas parturientes, podem ter sofrido viés de informação. Entretanto, buscar nas puérperas os motivos para o não tratamento dos parceiros fixos é de grande interesse e pode contribuir para o desenho de estratégias de intervenção, uma vez que demonstram a realidade e as dificuldades vivenciadas pelas mulheres. Destaca-se a baixa proporção de mulheres que foram diagnosticadas com sífilis no período gestacional e a dificuldade de captação e tratamento dos parceiros sexuais. Os parceiros são comunicados do diagnóstico de sífilis da gestante, entretanto, poucos são adequadamente tratados.

\section{Referências}

1. Brasil. Ministério da Saúde. Secretaria de Vigilância em Saúde. Programa Nacional de DST e Aids. Manual de controle das doenças sexualmente transmissíveis. 4a ed. Brasília (DF): Ministério da Saúde; 2006.

2. Brasil. Ministério da Saúde. Secretaria de Vigilância em Saúde. Programa Nacional de DST e Aids. Protocolo para a prevenção de transmissão vertical de HIV e sífilis. Brasília (DF): Ministério da Saúde; 2007.

3. Nascimento MI, Cunha AA, Guimarães EV, Alvarez FS, Oliveira SRSM, Villas Bôas EL. Pregnancies complicated by maternal syphilis and fetal death. Rev Bras Ginecol Obstet. 2012;34(2):56-62.

4. Brasil. Ministério da Saúde. Secretaria de Vigilância em Saúde. Programa Nacional de DST e Aids. Boletim Epidemiológico Aids e DST. Brasília (DF): Ministério da Saúde; 2007;4(1).

5. Pires ON, Pimentel ZNS, Santos MVS, Santos WA. Vigilância epidemiológica da sífilis na gravidez no centro de saúde do bairro Uruará-Área Verde. DST J Bras Doenças Sex Transm. 2007; 19(3/4): 162-5.

6. Lomotey CJ, Lewis J, Gebrian B, Bourdeau R, Dieckhaus K, Salazar JC. Maternal and congenital syphilis in rural Haiti. Rev Panam Salud Publica. 2009;26(3):197-202.

7. Brasil. Ministério da Saúde. Secretaria de Atenção à Saúde. Departamento de Ações Programáticas Estratégicas. Área Técnica de Saúde da Mulher. Pré-natal e puerpério: atenção qualificada e humanizada: manual técnico. Brasília (DF): Ministério da Saúde; 2006.

8. Donalísio MR, Freire JB, Mendes ET. Investigação da sífilis congênita na microrregião de Sumaré, Estado de São Paulo, Brasil - desvelando a fragilidade do cuidado à mulher gestante e ao recém-nascido. Epidemiol Serv Saúde. 2007; 16(3):165-73.

9. Almeida MFG, Pereira SM. Caracterização epidemiológica da sífilis congênita no município de Salvador, Bahia. DST J Bras Doenças Sex Transm. 2007;19(3/4): 144-56.

10. Fernandes RCSC, Fernandes PGCC, Nakata TY. Análise de casos de sífilis congênita na maternidade do Hospital da Sociedade Portuguesa de Beneficência de Campos, RJ. DST J Bras Doenças Sex Transm. 2007;19(3/4):157-61.

11. Figueiró-Filho EA, Gardenal RVC, Assunção LA, Costa GR, Periotto CRL, Vedovatte CA, et al. Sífilis congênita como fator de assistência pré-natal no município de Campo Grande - MS. DST J Bras Doenças Sex Transm. 2007;19(3/4):139-43.
12. Ramos Jr AN, Matida LH, Saraceni V, Veras MA, Pontes RJ. Control of mother-to-child transmission of infectious diseases in Brazil: progress in HIV/AIDS and failure in congenital syphilis. Cad Saúde Pública. 2007;23 (Suppl 3):S370-8.

13. Holanda MTCG, Barreto MA, Machado KMM, Pereira RC. Perfil epidemiológico da sífilis congênita no Município do Natal, Rio Grande do Norte - 2004 a 2007. Epidemiol Serv Saúde. $2011 ; 20(2): 203-12$

14. Melo NGDO, Melo Filho DA, Ferreira LOC. Diferenciais intraurbanos de sífilis congênita no Recife, Pernambuco, Brasil (2004-2006). Epidemiol Serv Saúde. 201 1;20(2):213-22.

15. De Lorenzi DRS, Madi JM. Congenital syphilis as a prenatal care marker. Rev Bras Ginecol Obstet. 2001;23(10):647-52.

16. Campos ALA, Araújo MAL, Melo SP, Gonçalves MLC. Epidemiologia da sífilis gestacional em Fortaleza, Ceará, Brasil: um agravo sem controle. Cad Saúde Pública. 2010;26(9):1747-55.

17. Mirkuzie AH, Hinderaker SG, Mørkve O. Promising outcomes of a national programme for the prevention of mother-to-child HIV transmission in Addis Ababa: a retrospective study. BMC Health Serv Res. 2010;10(9):267.

18. Figueiredo WS, Schraiber LB. Concepções de gênero de homens usuários e profissionais de saúde de serviços de atenção primária e os possíveis impactos na saúde da população masculina, São Paulo, Brasil. Ciênc Saúde Coletiva. 2011;16 (Supl 1): 935-44.

19. Sampaio J, Santos RC, Callou JLL, Souza BBC. Ele não quer com camisinha e eu quero me prevenir: exposição de adolescentes do sexo feminino às DST/aids no semi-árido nordestino. Saúde Soc. $2011 ; 20(1): 171-81$

20. Alam N, Streatfield PK, Shahidullah M, Mitra D, Vermund SH, Kristensen S. Effect of single session counselling on partner referral for sexually transmitted infections management in Bangladesh. Sex Transm Infect. $2011 ; 87(1): 46-51$.

21. Araújo CL, Shimizu HE, Sousa AIA, Hamann EM. Incidência da sífilis congênita no Brasil e sua relação com a Estratégia Saúde da Família. Rev Saúde Pública. 2012;46(3):479-86.

22. Capilheira MF, Santos IS. Fatores individuais associados à utilização de consultas médicas por adultos. Rev Saúde Pública. 2006;40(3):436-43.

23. Gomes R, Nascimento EF, Araújo FC. Por que os homens buscam menos os serviços de saúde do que as mulheres? As explicações 
de homens com baixa escolaridade e homens com ensino superior. Cad Saúde Pública. 2007;23(3):565-74.

24. Nascimento ARA, Trindade ZA, Gianordoli-Nascimento IF, Pereira FB, Silva SATC, Cerello AC. Masculinidades e práticas de saúde na região metropolitana de Belo Horizonte/MG. Saúde Soc. $2011 ; 20(1): 182-94$.

25. Araújo MAL, Leitão GCM. Acesso à consulta a portadores de doenças sexualmente transmissíveis: experiências de homens em uma unidade de saúde de Fortaleza, Ceará, Brasil. Cad Saúde Pública. 2005;21 (2):396-403.
26. Bertoni N, Bastos FI, Mello MB, Makuch MY, Sousa MH, Osis M, et al. Uso de álcool e drogas e sua influência sobre as práticas sexuais de adolescentes de Minas Gerais, Brasil. Cad Saúde Pública. $2009 ; 25(6): 1350-60$.

27. Araújo MAL, Silveira CB. Vivências de mulheres com diagnóstico de doença sexualmente transmissível - DST. Esc Anna Nery Rev Enferm. 2007; 11 (3):479-86.

28. Feitosa JA, Coriolano MWL, Alencar EM, Lima LS. Aconselhamento do pré-teste anti-HIV no pré-natal: percepções da gestante. Rev Enferm UERJ. 2010;18(4): 559-64. 